\title{
Nouveau Roman, Nouveau Monde ${ }^{1}$
}

\author{
Roger-Michel Allemand \\ Laboratoire Babel, Université du Sud-Toulon-Var
}

Toute fin est un commencement.

Sergiu Celibidache

Nouveau, ça ne veut rien dire. Preuve en est la facilité avec laquelle a été détourné le sens originel de l'expression «nouveau roman». En mars 1957, les éditions de Minuit publient une version revue et augmentée de Tropismes, de Nathalie Sarraute, initialement paru vingt ans plus tôt, en même

${ }^{1}$ Cet article est le texte d'une conférence donnée à Princeton University le 25 mars 2013. 
temps que le troisième roman d'Alain Robbe-Grillet, La Jalousie. C'est à cette occasion qu'Émile Henriot, «de l'Académie française », écrit dans Le Monde un article intitulé "Le nouveau roman ». Le texte est un éreintement et utilise l'expression de façon dépréciative, à la manière dont on avait jadis employé le mot «impressionnisme» (Leroy, 1874), pour dénigrer les nouveaux peintres de la pâte de Claude Monet, à partir du titre de son tableau Impression soleil levant. Jérôme Lindon et Alain Robbe-Grillet ont alors un coup de génie: tirer profit du retentissement de l'article d'Henriot pour créer de toutes pièces un mouvement littéraire encore inexistant en revendiquant l'étiquette Nouveau Roman, après l'avoir affublée de majuscules en guise de lettres de noblesse (voir Allemand, 2002).

Littérature : « Nouvelletés » de la Renaissance, Nouveau Roman, Nouveau Théâtre, New Narrative Poets... Humanités : Nouvelle Histoire, Nouvelle Critique, Nouvelle Philosophie, Nouvelle Sociologie... Beaux-Arts : Jugendstil, Art Nouveau, Nouveau Réalisme, Nouvelle Figuration... Musique : bossa nova, nuevo tango, New Beat, New Wave... Cinéma: Néo-réalisme, Nouvelle Vague... Politique: New Deal, New Labour... J'en passe.

France : Beaujolais Nouveau !

Non, décidément, nouveau, ça ne veut rien dire. Sauf pour le vin peut-être : à peine vendangé, déjà mis en bouteille. Son côté vert, son goût acidulé, son renom purement publicitaire. Produit de masse, aussitôt bu, aussitôt oublié. La cohésion sociale passagère, la communion de la jeunesse. Jeunesse du breuvage et verdeur des générations qui, toutes, un jour ou l'autre, ont dit aux Anciens : «Vous allez voir ce que vous allez boire! » 
«La nouveauté est une des conditions de l'enthousiasme», remarquait déjà Lamartine, en 1823... Avant-garde et modernité vont alors censément de pair. Dès la cristallisation de la mouvance néo-romanesque (voir Allemand, 1996), RobbeGrillet soulignait ainsi que «le roman depuis qu'il existe a toujours été nouveau » (1963, p. 10) et que «Flaubert écrivait le nouveau roman de 1860, Proust le nouveau roman de 1910 » (ibid.). Plus de cent ans auparavant, Stendhal nommait romanticisme tout art adapté aux besoins de son siècle, qui a le «courage » de "hasarder», qui ose «la manière d'étudier le monde au milieu duquel nous vivons » (1823), et rangeait donc Sophocle, Euripide, Racine, Shakespeare et Dante parmi les Romantiques de leurs temps - à leurs époques respectives.

Pour le reste, est évidemment nouveau ce qui n'est pas encore connu, ce qui étonne la réception, ce qui produit un changement radical - le fameux Horizontwandel théorisé par Jauss (1967) à partir de Gadamer (1960). Les Amériques n'étaient certes pas l'horizon d'attente de Christophe Colomb. De façon comparable, les lecteurs ne s'attendaient pas à la rénovation romanesque des années 1950. Dans les deux cas, on n'avait rien vu venir, on ne savait pas ce qui arrivait, on ignorait vers quoi l'on se dirigeait, personne n'était vraiment préparé.

\section{Expériences de la guerre}

Dans le champ littéraire, les perspectives étaient d'autant plus bouchées que l'horizon était recouvert des cendres de la Seconde Guerre mondiale. Les auteurs plus ou moins durablement associés à la mouvance néo-romanesque l'ont vécue de manière diverse. 
Alors que plusieurs écrivains et intellectuels quittent la France pour se réfugier à New York (André Breton, Antoine de Saint-Exupéry, Saint-John Perse, etc. - voir Mehlan, 2005), Samuel Beckett, lui, s'engage dans la Résistance dès 1940. Claude Mauriac rejoint les rangs de la France Libre et devient en 1944 le secrétaire particulier du général de Gaulle. Marguerite Duras concourt à l'activité d'un réseau de Résistance en 1943. Claude Simon, qui a brièvement pris part à la lutte des Républicains espagnols en 1936, est mobilisé trois ans plus tard dans la cavalerie; fait prisonnier, il s'évade en octobre 1940, pour regagner sa propriété en Zone non occupée. De confession israélite, Nathalie Sarraute est radiée du Barreau fin 1940, puis contrainte au divorce pour protéger son mari des lois antijuives de Vichy; elle se réfugie en 1942 dans l'écriture de Portrait d'un inconnu. En 1943-1944, Claude Ollier et Alain Robbe-Grillet effectuent ensemble le Service du travail obligatoire. De 1939 à 1944, Robert Pinget est mobilisé à la frontière de son pays natal, la Suisse. Quant à Michel Butor et Jean Ricardou, nés respectivement en 1926 et 1932, ils étaient encore trop jeunes pour participer à quoi que ce fût.

L'ayant néanmoins éprouvée dans sa chair, Butor parle de la Seconde Guerre mondiale, ce «temps de la faim, de la maigreur, de la peur et surtout du froid » (2009, p. 49), comme d' « une séparation encore bien plus grande que la précédente » (ibid.) : "J'en reviens toujours à la guerre car c'est une expérience fondamentale pour moi.» (1998, p. 259). Ollier abonde en ce sens lorsqu'il dit avoir vécu «le naufrage du continent [européen] en une quasi-destruction de ses forces et de ses valeurs » (1996, p. 36). Champ de ruines matérielles et culturelles donc. 


\section{In-scription du désastre}

L'art peut-il exister après l'Holocauste? On sait qu'Adorno (1981) répondait par la négative, du moins pour ce qui est de la poésie. Et pourtant... Claude Simon affirmait :

Si le surréalisme est né de la guerre de 1914, ce qui s'est passé après la dernière guerre est lié à Auschwitz. Il me semble qu'on l'oublie souvent quand on parle du «nouveau roman ». Ce n'est pas pour rien que Nathalie Sarraute a écrit L'Ėre du soupçon; Barthes, Le Degré zéro de l'écriture. Que des artistes comme Tapiès ou Dubuffet sont partis de graffitis, du mur, ou que Louise Nevelson a fait des sculptures à partir de décombres. Toutes les idéologies s'étaient disqualifiées. L'humanisme, c'était fini [...] : il n'y a plus de recours, essayons de revenir au primordial, à l'élémentaire, à la matière, aux choses. Exemple : Ponge. (1989)

Le "Générique » de Leçon de choses en fournit une illustration idoine, qui est jonché de «langues pendantes du papier décollé », de "plâtre humide et gris qui s'effrite, tombe par plaques », de "débris [...] éparpillés », d'« impalpable poussière blanche», de "carrelage [...] brisé», de "fragments», de " morceaux de bois, de briques, de vitres cassées », de " châssis démantibulé » (Simon, 1975, p. 9), etc. Duras précise quant à elle : «La guerre de 40 pour moi, sa spécificité unique, ce n'est pas l'ampleur des moyens mis en œuvre, c'est Auschwitz. » (1989). Et Robbe-Grillet de renchérir :

[...] c'est une véritable coupure que l'année 45 a représentée dans mon existence. Car mes rapports personnels avec l'ordre ont été profondément altérés à partir de la Libération, et surtout après l'entrée des troupes alliées en Allemagne, accompagnées chaque jour de monstrueuses révélations sur la matérialité des camps et sur toute la sombre horreur qui était la face cachée du national-socialisme. (1984, p. 122) 
Sans doute les trois auteurs eussent-ils pu ajouter à cette découverte de la barbarie l'autre apocalypse de la Seconde Guerre mondiale : l'invention de la bombe atomique. Ce n'est pas pour rien non plus que Duras a écrit le scénario et les dialogues d'Hiroshima mon amour (1960). Avec la découverte de la Solution finale, le 6 août 1945 marque en effet le terme d'une ère de foi inébranlable dans les vertus intrinsèques de l'humanité et ouvre une crise de civilisation reposant sur l'équilibre de la terreur, du moins jusqu'à la fin de la guerre froide qui s'ensuivit.

\section{Dé-scription de l'établi}

Autant dire que le Nouveau Roman procède de « l'Histoire avec sa grande hache » (Perec, 1975, p. 13). On comprend mieux, dès lors, qu'il fût tant marqué d'une atmosphère de chaos. Ainsi du Vent de Simon, dont un projet de sous-titre aurait précisé : "tentative de restitution d'un retable baroque d'après les fragments retrouvés dans une chapelle en ruine» (RobbeGrillet, dans Allemand, 2002, p. 334, n. 43). De fait, l'une des principaux traits de la création postmoderne ressortit à une esthétique $\mathrm{du}$ fragment. Ce roman évoque une tornade originelle, "force déchaînée, sans but, condamnée à s'épuiser sans fin, sans espoir de fin» (Simon, 1957, p. 241). De façon comparable, La Route des Flandres détaille la décomposition, dans un contexte de débâcle généralisée, «comme si non pas une armée, mais le monde lui-même tout entier et non pas seulement dans sa réalité physique mais encore dans la représentation que peut s'en faire l'esprit [...] était en train de se dépiauter se désagréger s'en aller en morceaux en eau en 
rien» (1960, p. 16). Ainsi encore, chez Duras, de la digue dérisoire d'Un barrage contre le Pacifique (1950) ou de L'Amant, où l'auteur dresse d'elle-même un autoportrait en ruine, le «visage détruit» (1984, p.10) et représente les épaves charriées par les flots du Mékong :

[...] le fleuve [...] a ramassé tout ce qu'il a trouvé depuis [...] la forêt cambodgienne. Il emmène tout ce qui vient, des paillotes, des forêts, des incendies éteints, des oiseaux morts, des chiens morts, des tigres, des buffles, noyés, des hommes noyés, des leurres, des îles de jacinthes d'eau agglutinées, tout va vers le Pacifique, rien n'a le temps de couler, tout est emporté par la tempête profonde et vertigineuse du courant intérieur, tout reste en suspens à la surface de la force du fleuve. (1984, p. 30)

Belle image de l'inspiration durassienne qui crée à partir de la dévastation. Détruire, dit-elle (Duras, 1969)... On n'est pas loin ici de la menace du pourrissement chez Ollier, en particulier dans Été indien, qui est... « un livre sur New York » $(1996$, p. 33) :

On imaginerait un monde chaotique, des terres bourbeuses, la jungle, l'eau des fleuves charriant des bêtes mortes, charriant des souches, des palmes, des fleurs rouges, l'eau s'infiltrant, montant sous les racines, l'eau stagnante. (Ollier, 1963, p. 7)

Ainsi enfin de Pinget, dont l'œuvre évoque maints décombres et délabrements, entre autres dans Fable — « La ville avait fondu sous l'effet d'un cataclysme, il n'en restait que des scories. » (Pinget, 1971, p.10) - , ou de Robbe-Grillet, qui vante une poétique de la ruine, des fragments et des débris à la dérive, dès Un régicide (écrit en 1949), dont le château délabré est sur le point de s'écrouler, édifice des anciens systèmes de valeurs, désormais périmés -, comme chez Butor, la maison de Passage de Milan est bâtie sur une ancienne crypte d'église effondrée. Le premier titre de ce premier roman était d'ailleurs L'Entrepôt, ce qui montre bien le changement d'état d'esprit. 
Pour autant, le constat n'est pas forcément désespéré. Simon avance même que

les ruines sont des manifestations de la vie dans ce qu'elle a de plus robuste, et tout passé est une addition de ruines auxquelles le temps, les mutilations, confèrent une majesté durable que l'édifice ainsi ennobli n'avait pas à l'état neuf. Nous sommes tous constitués de ruines: celles des civilisations passées, celles des événements de notre vie dont il ne subsiste dans notre mémoire que des fragments. (Simon, 1988a, p. 18)

De la «Cendre» (Simon, 1959) aux «Matériaux de construction » (1960b) en somme. Dans une autre perspective, Robbe-Grillet vante aussi les ressources de la catastrophe, qui est appel à une relance. En témoigne La Reprise (2001), dont l'action se situe dans le Berlin de l'après-guerre. Quelques années plus tôt, l'auteur revendiquait :

l'ancienne suprématie du sens [...] nous a laissé l'esprit dans une joyeuse dérive : [...] les significations parcellaires et les signes égarés ayant perdu de proche en proche leurs cohésions œcuméniques, leur solidarité totalitaire, se sont désintégrés bientôt en un grouillement de particules élémentaires de plus en plus fines, qui se déplacent maintenant sans repos en quête de possibles assemblages, aléatoires, instables, dans une immense production d'énergie dépourvue de finalité. (1994, p. 146)

Le passage suscite la figure de Joyce - Robbe-Grillet y fait d'ailleurs explicitement référence au moment de La Reprise -, aux quarks tourbillonnants de Finnegans Wake, qui, dès 1939, prenait acte des vertigineux mouvements de la physique des particules. Et nul doute qu'on ne rencontre des grouillements similaires dans les remous des profondeurs qui animent la sousconversation de Nathalie Sarraute (voir 1957), à savoir un

foisonnement de sensations, d'images, de sentiments, de souvenirs, d'impulsions, de petits actes larvés qu'aucun langage intérieur n'exprime, qui se bousculent aux portes de la 
conscience, s'assemblent en groupes compacts et surgissent tout à coup, se défont aussitôt, se combinent autrement et réapparaissent sous une autre forme. (Sarraute, 1956, p. 96-97)

Métaphore. Déplacement. Ailleurs.

\section{Des Européens en Amérique}

Dans l'immédiat après-guerre, les États-Unis sont un pôle d'attraction, ainsi que Butor le résume :

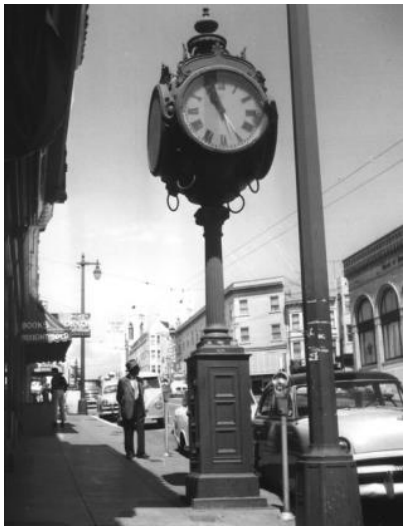

Photo prise par Butor aux USA dans les années 1960
Confusément, sur les jeunes intellectuels que nous étions, l'Amérique ce n'était plus seulement l'avenir mais c'était aussi une nouvelle Histoire. (1998, p. 250)

Nous avons eu le sentiment très net que des nouveautés esthétiques capitales venaient depuis l'autre côté de l'Atlantique. [...] Nous avions très fort le sentiment, lorsque l'un d'entre nous revenait des États-Unis, qu'il était en avance - en ce qui concernait les arts mais aussi la vie quotidienne. (ibid., p. 251)

Que l'on pense à la revendication d'une identité picturale américaine, dès 1945, par exemple dans le documentaire qui suit la projection du film tiré du roman d'Oscar Wilde: The Picture of Dorian Gray, réalisé par Albert Lewin (MGM). Il s'agit d'un court métrage de 1944 où l'on voit les frères Albright peindre les deux tableaux destinés au film; il est intitulé Grandpa Called It Art, ce qui tourne en dérision l'admiration américaine pour les Beaux-Arts européens. 
Sur le plan du dialogue des arts justement, Robbe-Grillet s'est très tôt intéressé à l'expressionnisme abstrait - Jackson Pollock, Mark Rothko et Willem de Kooning (voir 1984, p. 194) -, au Pop Art de Jasper Johns (1978b) et de Robert Rauschenberg (1978c), avec lesquels il travailla. Mark Tansey (cf. 1993 et 1994, p. 152) et George Segal (cf. 1990 et 1994, p. 169-178) étaient également de ses amis. Il avait lui-même un atelier à New York et s'est servi de certaines œuvres de ces peintres américains comme d'«incitateurs iconiques» (voir Morrissette, 1978 et 1979).

De même, Simon se sentait «très près » (1988b, p. 180) de Rauschenberg - si près que le point de départ pour Les Corps conducteurs, et donc Orion aveugle, a été le tableau intitulé Charlene. Voici ce qui l'y avait touché :

La composition, la combinaison de ces matériaux «bruts », des bouts de tissu, la peinture dégoulinante, des photos ou des reproductions commerciales d'œuvres d'art, parfois voilées d'un glacis, des morceaux de bois, etc. (Simon, 1988b, p. 180)

De son côté, Butor a écrit sur Rothko (1968) et Johns (cf. Collectif, 1992 et Butor, 2007), et son Mobile est dédié : «À la Mémoire de Jackson Pollock ».

Lauréats de la Fondation Ford, Ollier et Pinget viennent en Amérique fin 1959. L'année suivante, Butor traverse également l'océan Atlantique, sur le paquebot United States. La nouveauté qui frappe ces Européens, impréparés au gigantisme du continent et à l'American Way of Life, est aussi diverse que leur personnalité. L'attention d'Ollier est retenue par les «fumerolles de Times Sq. : celles des regards des égouts et des canalisations de chauffage» (1984, p.132), l'ascenseur de l'Empire State Building qui «passe en quelques secondes du 
quatre-vingtième étage au rez-de-chaussée » (p. 136), l'horloge "plaquée dans le trottoir, incrustée dans la pierre » (p.143), «les avertisseurs stridents, perçants, interminables, des voitures de police, suivis des cloches des pompiers » (p. 152), «l'immense écran cinémascopique» (p. 180) du drive-in — et même par le métro new-yorkais qui «passe brusquement sous terre et ressort de façon très inattendue» (p. 134). Ces observations se retrouveront, modifiées, retravaillées, dans Été indien. Dans le même ordre d'idées, Butor retiendra les autoroutes, les supermarchés - et « ces petites charrettes, inventées pendant la guerre dans l'Oklahoma et qui maintenant se sont répandues dans le monde entier : le caddy » (1998, p. 252).

Pour ce qui est de Robbe-Grillet, quand il débarque à New York, c'est l'escalier de secours extérieur aux immeubles, typique des grandes villes nord-américaines, qui le retient plus particulièrement. On le retrouve dans Projet pour une révolution à New York: " escalier de fer extérieur, prévu comme descente de secours en cas de sinistre: squelette de lignes noires entrecroisées qui dessine des $\mathrm{Z}$ superposés de haut en bas de chaque immeuble, s'arrêtant toutefois à trois mètres du sol. Une mince échelle amovible, habituellement relevée, complète l'ensemble pour faire le raccord avec la chaussée, et permettre de fuir l'incendie qui embrase l'escalier intérieur » (1970, p. 14-15). Le roman est aussi marqué par la variété ethnique consubstantielle au melting-pot : indiens, blancs, noirs, davantage encore de métis (passim). (Notons que, près de dix ans plus tôt, dans son premier livre inspiré des États-Unis, Butor avait déjà joué sur les trois premières couleurs de peau du peuplement américain : rouge indien, blanc européen, noir africain.) 
Quant à Simon, il retrouve dans le bourgeonnement du skyline new-yorkais l'expression urbaine d'un élan vital, immaîtrisé, d'une course désordonnée, d'une sorte de compétition "naturelle», comme un acrotropisme dont le désordre apparent et la «beauté convulsive» (Breton, 1992, p. 687) pourraient s'apparenter à une « explosante-fixe » (ibid.) :

Aucune ville ne répond mieux à l'expression «sortie de terre » que New York (ou faudrait-il plutôt dire « jaillie») : et non pas exactement debout, statique, mais explosant, toujours en expansion non pas en surface mais en hauteur comme on peut voir sur certaines photographies prises d'avion (ou d'hélicoptère) avec un objectif grand angle (fish-eye), quand, bien sûr pendant une fraction de seconde, elle semble restée immobile alors qu'en fait elle n'a cessé de croître, de s'élever, ce genre d'objectifs exagérant la perspective, de sorte que ses multiples gratte-ciel apparaissent non pas verticaux, parallèles, mais obéissant à une force divergente, faisant penser à ces gerbes de cristaux allant s'écartant, se bousculant, poussant vers le ciel ses tours de toutes hauteurs, les moins élevées non pas résignées à leur sort mais ayant simplement pris leur essor avec un peu de retard et se dépêchant pour rattraper les autres, l'ensemble comme planté sur la rotondité de la terre à partir d'une étroite base comme une sorte d'explosion solidifiée, de phénomène naturel, anarchique, tumultueux et géométrique. (Simon, 1997, p. 284)

Entre 1964 et 1995, Sarraute séjourne une quinzaine de fois aux États-Unis. En 1972, en particulier, elle présente trois conférences à Princeton University. Sur le plan personnel, contrairement au snobisme français de ses contemporains (voir Bouchardeau, 2003, p. 205-207), elle s'enthousiasme aussitôt pour les mégalopoles, pour l'architecture verticale de New York et plus encore pour la variété stylistique de celle de Chicago: l'architectonique de Frank Lloyd Wright ou de Graham 
Anderson, les gratte-ciel de Solon Spencer Bernan, de William Le Baron Jenney, de Burnham aussi.

Elle n'est pas la seule à revenir. En 1970, Ollier effectue ainsi une tournée de conférences dans le Middle West. Pour ce qui est de Pinget, il retourne trois fois aux États-Unis, « pour des conférences, des lectures et une belle représentation d'Architruc » (Pinget, 1993, p. 197), et conservera toute sa vie une prédilection pour New York: "Que de bons amis là-bas, que de prévenance envers moi. Quant à la ville j'en aurais bien fait ma troisième patrie... On y respire la liberté comme nulle part ailleurs. » (ibid.).

Cet attrait ne tient pas uniquement aux invitations dont les Nouveaux Romanciers ont bénéficié. Le fait d'importance est que les universités américaines leur ont tout de suite reconnu des compétences professorales que la France leur déniait, en raison même de son fonctionnement administratif dans le Supérieur: pour enseigner la littérature, il ne faut pas être écrivain, il faut être titulaire d'un diplôme de littérature. Or Duras, Cl. Mauriac, Ollier, Pinget, Sarraute ont suivi des études de Droit; Robbe-Grillet était ingénieur agronome; Simon, seulement bachelier, section mathématiques élémentaires, a suivi ensuite des cours de peinture! Seul Butor, en définitive, était « qualifié » pour enseigner à l'Université. Pourtant, malgré sa thèse de doctorat, brillamment soutenue en 1973, l'inscription sur la liste d'aptitude lui a été refusée par le Comité consultatif compétent, pour non-conformité dans les étapes habituelles de la carrière.

Les États-Unis lui avaient heureusement ouvert les bras dès 1960 : outre les conférences qu'il donna dans tout le pays lors de ce premier séjour, il y enseigna en Pennsylvanie et dans 
le Vermont, puis à Evanston (1965), New York (1967) et Albuquerque (1967 et 1973), en tant que Visiting Professor. Quant à Robbe-Grillet, découvert par le professeur Morrissette, qui l'a fait connaître aux États-Unis et auquel il y doit la part initiale de son renom, il a enseigné à New York (1972-1997), Los Angeles (1977), Edmonton (1983), Gainesville (1985), Davis (1986), Saint-Louis (1986-1992) et Greensboro (1987).

Parmi les nombreux événements qui ponctuèrent l'itinéraire au long cours du Nouveau Roman de ce côté de l'Atlantique, relevons le colloque Three Decades of the French New Novel, en 1982, à New York University, en présence de Pinget, Robbe-Grillet, Sarraute et Simon (voir Oppenheim, 1986). Et c'est encore à New York que, l'année suivante, Sarraute et Robbe-Grillet interprétèrent ensemble, en compagnie d'Eugène Ionesco, Freshwater de Virginia Woolf.

On pourrait sans doute égrener davantage encore le détail de telles circonstances, mais cela n'aurait guère d'intérêt savant. Le point crucial est que les États-Unis ont joué un rôle déterminant dans la diffusion internationale du Nouveau Roman. À titre d'anecdote personnelle, je me rappelle ainsi que lorsque l'académie Nobel a décerné son prix à Claude Simon, j'ai entendu dire par un esprit pourtant éclairé : «Ah oui, celui qui fait des phrases de plusieurs pages... » Cette condescendance est assez représentative du climat intellectuel français de l'époque, alors qu'en Amérique, le Nouveau Roman était un objet d'étude universitaire et un sujet littéraire vedette depuis déjà une vingtaine d'années. 


\section{S'in-spirer de l'Amérique}

Le plus intéressant consiste à essayer de déterminer en quoi la découverte du Nouveau Monde a pu infléchir, ou même influencer, l'écriture de tel ou tel Nouveau Romancier. Sur ce point, je voudrais tout d'abord résumer les apports de Christian Milat, qui a comparé la manière dont New York a été rendue fictive par Ollier, dans Été indien, et par Robbe-Grillet, dans Projet pour une révolution à New York (voir Milat, 2006).

Ollier a effectué d'«innombrables et interminables parcours à pied dans les différents quartiers de la ville » (1996, p. 41). Le travail du romancier consiste, après un laps de temps indéfini, à réactiver ses sensations, ses perceptions, afin d'aboutir à la «re-création des lieux par l'écriture » (p. 53), si bien que «[l]'itinéraire du protagoniste dans New York [...] draine, chemin faisant, toutes les données et les composantes historiques, économiques, linguistiques, sociales, du pays » (p. 141). Pourtant, le nom de la ville n'est jamais cité. Ce n'est pas un oubli, ni un lapsus, ni un acte manqué. L'omission est volontaire, car, selon le Nouveau Romancier, donner les toponymes réels reviendrait non seulement sacrifier à l'ordre de la représentation référentielle, mais serait aussi, voire surtout, "convoquer dans la fiction toute la mythologie attachée à ces appellations et contrarier d'autant [s]es efforts pour faire sentir la réalité vivante, véritable de ces villes, loin des idées reçues » (p.134). Il s'agit en somme de favoriser un nouveau réalisme, qui n'est pas dans la tentative de restitution mimétique du monde, mais dans la saisie phénoménologique de l'expérience vécue, en s'efforçant de " "coller" au plus près des impressions fortes éprouvées par le nouveau venu dans cet 
univers exotique où il est plongé presque sans transition » (p. 22), pour mieux « remett[re] en cause, par les moyens de la langue et des récits, les données même de ce monde » (p. 141).

À l'inverse d'Ollier, Robbe-Grillet n'efface pas le mythe new-yorkais : il l'utilise pleinement et dans le sens de la plus grande stéréotypie possible, tel un réservoir de matériaux. Ces générateurs de texte choisis «au sein de quelques objets mythologiques contemporains » $(1972$, p. 160) — « couvertures illustrées des romans qu'on vend dans les gares, affiches géantes, revues pornographiques des sex-shops, publicités vernies des magazines de mode, figures peintes à plat des bandes dessinées » (p. 161) —, permettent une distanciation paradoxale, qui vise à neutraliser la force potentiellement mortifère de ces «éléments mythologiques » ainsi que leur charge dans l'inconscient collectif, en les déconstruisant et les subvertissant au profit de la «liberté » du créateur et de son imagination. Notons d'ailleurs que New York est aussi présente chez Claude Simon pour évoquer un magasin de revues pornographiques (voir Simon, 1971)...

La grande ville américaine est évidemment un lieu privilégié [...], en raison de la charge mythologique particulièrement violente qu'elle charrie, tant dans notre esprit d'Européens que dans celui des New-yorkais eux-mêmes. Ville imaginaire s'il en fût, elle représente un paroxysme jamais atteint de merveilles et de terreurs, avec son monde underground du crime, du vice, de la drogue, matérialisé par le réseau immense et délabré du métro souterrain. (Robbe-Grillet, 1970b, p. 3-4)

The Empire City est en effet ici « une ville parfaitement imaginaire » (Robbe-Grillet, 1972, p. 166) : « [...] personne n'a jamais prétendu que le récit était fait par un Américain. » (1970a, p.189), précise le texte du roman. Et son auteur de 
confirmer : " Il suffit d'aller à New York pour constater que les avertisseurs des pompiers ne font pas du tout le même bruit que dans Projet pour une révolution à New York, et que le métro qui joue un rôle si important dans mon roman n'est autre en réalité que celui de Paris. » (1976).

Alors quid de «la fiction de l'Amérique» (Baudrillard, 1986, p. 33) ? Tout se résumerait-il à l'opposition facile entre le système consumériste et le modèle altruiste, entre « l'Amérique sidérale, celle de la liberté vaine et absolue des freeways» (p. 10), et «celle du social et de la culture » ? Certes non (voir Pinçonnat, 2001).

\section{Vivre l'Amérique}

Le cas de Butor mérite qu'on s'y attarde davantage, pour le retentissement de la découverte des États-Unis sur son œuvre - et il est immense : « un choc considérable » (1998, p. 251). Quand il en parle, Michel rappelle toujours qu'après la Seconde Guerre mondiale, le voyage outre-Atlantique était presque un passage obligé pour un intellectuel européen et le rattache à la tradition littéraire des voyages en Italie et en Orient, au XIX siècle. Plus même, pendant l'année universitaire 1974-1975, l'écrivain a donné un cours intitulé « Représentation des ÉtatsUnis dans la littérature française », où il examine la transformation de l'image du pays depuis Chateaubriand jusqu'à Céline, en passant par Alexis de Tocqueville, Charles Baudelaire, Victor Hugo, Villiers de l'Isle-Adam, Jules Verne, Paul Claudel, Guillaume Apollinaire ou Blaise Cendrars. Et Butor a le sentiment qu'il s'inscrit lui-même dans cette continuité 
historique. Ce n'est donc pas tant the American Dream qui le préoccupe que son devenir :

Dans Mobile, j'ai fait rêver les citoyens des États-Unis, en faisant passer le rêve de lieu en lieu, ce qui fait que c'est le même rêve qui se poursuit, mais le sujet rêvant se trouve dans un État ou dans un autre; à la tombée de la nuit, les rêves balaient le continent. (Butor, 2006b, p. 720)

Dans USA 76 (Bicentenaire Kit), réalisé à l'occasion de la célébration de la Déclaration d'Indépendance (texte repris dans Butor, 1978), blues et rêve(s) sont répartis symétriquement...

À l'époque de son premier séjour sur le sol américain, Butor vient d'achever son quatrième roman, Degrés (1960), où il a poussé la complexité narrative amorcée auparavant. La découverte des États-Unis lui est une véritable secousse, d'abord dimensionnelle. Il se dit que désormais, il ne pourra plus écrire de roman; le genre est trop limité, ne recèle pas assez de ressources pour contenir, transcrire toute la richesse et la diversité de son expérience :

Aux États-Unis le paysage m’a énormément frappé, [...] là, le paysage s'étend dans toutes les directions, avec une variété d'organisation de l'espace extraordinaire et très difficile à saisir. Dans l'est du pays, cette organisation est encore européenne. Dans le Middle-West, c'est profondément différent, c'est le règne de l'angle droit. Et puis il y a les déserts, et les Indiens qui y vivent, la façon dont ils les habitent, leur perception de l'espace qui ne se limite pas aux quatre points cardinaux, mais qui comprend aussi le zénith et le nadir, sous d'autres appellations. J'ai dû adapter mes instruments. (Butor, 2009, p. 105)

Cela donnera Mobile. Étude pour une représentation des ÉtatsUnis. Butor découvre et se découvre autre, tel Colomb face à «l'existence même de ce continent, surgi d'au-delà de l'horizon 
[...] là où il n'aurait pas dû être... » (2009, p. 107). L'imaginaire "d'une seconde naissance» (2006b, p. 514) prend également une dimension collective, ainsi qu'il l'écrit en 1964 :

L'Amérique doit être le bain de Jouvence qui rajeunira notre civilisation vieillie, qui nous permettra de retrouver la jeunesse de ce que nous ne connaissons, dans notre civilisation, que vieilli. (Butor, 2006b, p. 514)

Et cela passe, comme de juste, par la redécouverte de la sauvagerie précolombienne, du naturel originaire, des Native Americans :

Ces civilisations indiennes, rajeunies jusqu'à la veille de leur mort par le bain de Jouvence qu'est le continent américain, le «désert», ces civilisations qui meurent écrasées par la «vieille» Europe, que contenaient-elles en puissance? Quel héritage en pouvons-nous recevoir? (Butor, 2006b, p. 514)

En réponse à lui-même, par le biais de Chateaubriand, l'auteur avance que "peut-être que grâce aux conditions naturelles particulières du continent américain, cette civilisation aurait réussi ce miracle de s'accroître sans vieillir, sans perdre son harmonie, sans qu'intervienne cette scission fatale entre nousmêmes et le reste du monde » (Butor, 2006b, p. 515), avant de poursuivre : «[...] cette aurore si rapidement transformée en crépuscule, il faut en conserver l'éclat, il faut qu'un Européen "renaisse" Indien, et fasse entendre cette voix, fasse entendre cette protestation d'une race abolie, cette vérité, ce "génie"; le texte alors lui-même deviendra fontaine de Jouvence. » (ibid.)

Première remarque: la proximité intellectuelle de ces lignes avec Saint-John Perse ; avec ce qu'il écrivait depuis son exil aux États-Unis (1940-1958), c'est-à-dire au cours de la période la plus féconde de son œuvre, après vingt-cinq ans de silence poétique: «Le vrai drame du siècle est dans l'écart 
qu'on laisse croître entre l'homme temporel et l'homme intemporel. » (Perse, 1982, p. 446), et c'est la crise de l'esprit qui fait la crise de civilisation. Fasciné par le Nouveau Monde, loin du désenchantement et du pessimisme ambiants de l'Ancien, revendiquant le décalage comme identité même, Perse entendait en effet fonder un humanisme visionnaire et volontariste, proposé comme un antidote au désespoir issu du désastre que vivait le continent européen. À l'âge désormais nucléaire, où la fission de l'atome avait débouché sur l'explosion des valeurs humanistes et la destruction du monde (voir Raybaud, 2005), le lauréat du prix Nobel 1960 proposait un recentrement sur l'homme, une ascèse poétique qui permît de retrouver le noyau insécable des origines. Saint-John Perse n'était pas un Nouveau Romancier, bien entendu, mais, justement, la remise en perspective et le Zeitgeist permettent de relativiser le point de vue.

Deuxième remarque: Butor inscrit ici le continent américain dans le champ de l'utopie régressive. Zeitgeist, disaisje à l'instant: non plus le génie du christianisme, ni même le génie du lieu, mais le génie du temps. D’un temps arrêté, où le vieillissement n'existerait pas, ni ses effets, ni la mort. De l'Amérique donc, comme une néguentropie. Et il n'est pas fortuit que l'un des trois livres américains de Butor, Oǔ, écrit alors qu'il vivait au Nouveau-Mexique évoque les cérémonies des Zunis, qui forment un rite de recréation du monde, de renouvellement des énergies, de renaissance de soi :

En effet, la marge, la frange, la « frontière » au sens de l'histoire des États-Unis ; le Far West était un refuge pour la liberté, un lieu d'invention, un creuset d'émergence pour une vérité nouvelle. (Butor, 2009, p. 32) 
Oǔ est en effet l'accomplissement d'une trilogie qui ne dit pas son nom. L'accent du mot est barré : le lieu est tout à la fois biffé et signalé, comme d'une croix sur une carte au trésor. Le sens devient indécidable: ni pronom relatif, ni conjonction de coordination. Ni situation dans l'espace, ni lien syntaxique, ni alternative. Oú, c'est autre chose, un troisième terme, plus qu'un néologisme, un barbarisme volontaire, une extranéité qui fait hapax. L'oblique du signe diacritique marque le terme d'une étape de dix ans, le terminus postquem d'un parcours entamé avec Mobile et poursuivi par 6810000 litres d'eau par seconde : après l'horizontalité de la traversée des États-Unis, après la verticalité des chutes du Niagara, la diagonale du Z des Zunis et si vous me permettez le mauvais jeu de mots, des États-Unis, dans la transversalité, la diagonale. Et l'on se prend à songer à ce que Butor écrit à propos d'Apollon : «Il est Loxias, c'est-àdire l'oblique, l'énigmatique, celui par qui l'énigme prend forme, au lieu de demeurer illimitée, contagieuse et destructrice. » (Butor, 2007b, p. 55)

Pareil dans Mobile: c'était très important que ça ne soit pas seulement mon voyage aux États-Unis, l'opinion que j'en ai, non, je voulais absolument trouver le moyen de faire parler les États-Unis. D'où tous les personnages que j'ai cités textuellement dans le livre, citations qui ont tellement surpris ceux qui connaissaient ces classiques américains incomparablement mieux que moi. D'un autre côté, ce biais ne m'empêchait pas de parler de moi aux États-Unis, puisque, à partir du moment où j'y étais, j'en devenais une partie, même infime, et j'en modifiais d'une certaine manière la structure. (Butor, 2009, p. 64)

Depuis ses premiers pas aux États-Unis, Butor n'a cessé de le faire, puisqu'il y a encore séjourné pour son plaisir en 1995, 2002 et 2012. 
Ich bin ein Berliner, proclamait le président Kennedy. À quoi je répondrais ce que nous savons tous déjà : We are all Americans. Sans doute plus que jamais depuis le 11-Septembre. Aussi emprunterai-je à Robbe-Grillet les presque mots de la fin : «Ai-je signalé que, même avant la révolution, toute la ville de New York, et en particulier l'île de Manhattan, était depuis longtemps en ruines? Je parle bien entendu des constructions de surface, à l'air prétendu libre. » (Robbe-Grillet, 1970a, p. 207).

Avant lui, Paul Valéry postulait que «le temps du monde fini commence » (1960, p. 923). Simon lui faisait écho, lorsqu'il observe : «il peut même arriver qu'à la "fin" on se retrouve au même endroit qu'au "commencement"». De nos jours, un nouveau roman émerge du Nouveau Monde, il est issu de Ground Zero.

\section{Bibliographie}

ADORno, Theodor. (1981), Prisms, Cambridge, MIT Press : "Cultural Criticism and Society" (1951).

Allemand, Roger-Michel. (1996), Le Nouveau Roman, Paris, Ellipses, coll. «Thèmes \& études »;

- . (2002), « Robbe-Grillet à Minuit : editoring et lancement du Nouveau Roman (1955-1963) », dans François BESSIRE (dir.), Travaux de littérature, $\mathrm{n}^{\circ} 15$ : "L'Écrivain éditeur. 2. XIXe et XXe siècles”, Boulogne, ADIREL, septembre, p. 319-348.

BAUdrillard, Jean. (1986), Amérique, Paris, Grasset. 
BouchardeaU, Huguette. (2003), Nathalie Sarraute, Paris, Flammarion, coll. « Grandes biographies ».

Butor, Michel. (1954), Passage de Milan, Paris, Minuit; repris dans Butor, 2006a, p. 55-217 ;

—. (1958), Le Génie du lieu, Paris, Grasset, coll. « La Galerie» ; repris dans Butor, 2007b, p. 29-109;

—. (1960), Degrés, Paris, Minuit; repris dans Butor, 2006a, p. 681-1001;

- (1962), Mobile. Étude pour une représentation des États-Unis, Paris, Gallimard ; repris dans Butor, 2007b, p. 115-419 ;

-. (1964), "Chateaubriand et l'ancienne Amérique», dans Répertoire II, Paris, Minuit, coll. «Critique» ; repris dans Butor, 2006b, p. 491-524 ;

-. (1965), 6810000 litres d'eau par seconde. Étude stéréophonique, Paris, Gallimard ; repris dans Butor, 2007b, p. 601-899;

—. (1968), «Les Mosquées de New York ou l'art de Mark Rothko », dans Répertoire III, Paris, Minuit, coll. « Critique»; repris dans Butor, 2006b, p. 1003-1018;

-. (1971), Oǔ. Le Génie du lieu 2, Paris, Gallimard ; repris dans Butor, 2007c, p. 27-414 ;

-. (1976), USA 76 (Bicentenaire Kit), en collaboration avec Jacques MonORY, Paris, Le Club du livre ;

—. (1978), Boomerang. Le Génie du lieu 3, Paris, Gallimard; repris dans Butor, 2007c, p. 419-876; 
-. (1982), "La Langue de l'exil », dans Répertoire V, Paris, Minuit, coll. «Critique » ; repris dans Butor, 2006b, p. 719721 ;

—. (1998), "Où les Amériques commencent à faire Histoire », dans Mireille Calle-Gruber (dir.) Butor et l'Amérique, Paris, L'Harmattan, coll. « Trait d'union », p. 249-260 ;

—. (2006a), OEuvres complètes, Paris, La Différence, t. I ;

—. (2006b), CEuvres complètes, Paris, La Différence, t. II ;

—. (2006c), CEuvres complètes, Paris, La Différence, t. III ;

—. (2007a), Comment écrire pour Jasper Johns, Paris, Notari ;

—. (2007b), CEuvres complètes, Paris, La Différence, t. V ;

—. (2007c), CEuvres complètes, Paris, La Différence, t. VI ;

-. (2009), Michel Butor. Rencontre avec Roger-Michel Allemand, Paris, Argol, coll. « Les Singuliers ».

Clergue, Yolande (dir.). (1992), Jasper Johns. Gravures et dessins de la collection Castelli, 1960-1991, préface de Lucien WEYGand et Marcelin PLEYNET, textes de Michel Butor et de Kathleen SLAVIN, Arles, Fondation Vincent van Gogh.

DURAS, Marguerite. (1950), Un barrage contre le Pacifique, Paris, Gallimard ;

-. (1960), Hiroshima mon amour, Paris, Gallimard ;

—. (1969), Détruire, dit-elle, Paris, Minuit ;

—. (1984), L'Amant, Paris, Minuit ;

-. (1989), entretien avec Colette MAZABrard, Cahiers $d u$ cinéma, $\mathrm{n}^{\circ} 426$, décembre. 
GADAMER, Hans Georg. (1960), Wahrheit und Methode. Grundzüge einer philosophischen Hermeneutik, Tübingen, Mohr Verlag. (trad. fr. : Vérité et méthode, Paris, Seuil, 1996).

HENRIOT, Émile. (1957), «Le Nouveau roman: La Jalousie, d'Alain Robbe-Grillet, Tropismes, de Nathalie Sarraute », Le Monde, 22 mai, p. 8-9.

JAUSS, Hans Robert. (1967), Literaturgeschichte als Provokation, Frankfurt, Suhrkamp, p. 144-208 (trad. fr. Pour une esthétique de la réception, Paris, Gallimard, 1978, p. 21-80).

JoYCE, James. (1939), Finnegans Wake, Londres, Faber \& Faber.

LAMARTINE, Alphonse de. (2006 [1823]), Méditations poétiques. Nouvelles méditations poétiques, Paris, Librairie Générale Française, coll. «Le Livre de Poche / Les Classiques de Poche » : «Préface des Secondes Méditations ».

LEROY, Louis. (1874), "L'Exposition des impressionnistes », Le Charivari, 25 avril.

MEHLMAN, Jeffrey. (2005), Émigrés à New York. Les intellectuels français à Manhattan 1940-1944, Paris, Albin Michel.

MiLAT, Christian. (2006), «Quand Ollier et Robbe-Grillet fictionnalisent New York», dans Amaryll CHANADY, George HANDLEY et Patrick ImBert (dir.), Les Mondes des Amériques et les Amériques du monde / Americas'Worlds and the World's Americas, Ottawa, Legas, p. 419-429.

MorRISSETTE, Bruce. (1978), «Intertextual Assemblage as Fictional Generator: Topologie d'une cité fantôme», The International Fiction Review, vol. 5, no 1, janvier, p. 1-14 ; 
-. (1979), Intertextual Assemblage in Robbe-Grillet, from "Topology" to "The Golden Triangle", Fredericton, York Press.

OLLIER, Claude. (1963), Été indien. Paris, Minuit ;

—. (1984), Cahiers d'écolier (1950-1960), Paris, Flammarion, coll. «Textes »;

-. (1996), Cité de mémoire. Entretiens avec Alexis Pelletier, Paris, P.O.L.

OpPEnHEIM, Lois (dir.). (1986), Three Decades of the French New Novel: Proceedings of a Colloquium Held in the Fall of 1982 at New York University, Urbana, University of Illinois Press.

PEREC, Georges. (1975), W ou le souvenir d'enfance, Paris, Denoël.

PinçonNat, Crystel. (2001), New York, mythe littéraire français, Genève, Droz.

PINGET, Robert. (1971), Fable, Paris, Minuit ;

—. (1973), Paralchimie, suivi de Architruc, L'Hypothèse, Nuit, Paris, Minuit ;

-. (1993), Robert Pinget à la lettre. Entretiens avec Madeleine Renouard, Paris, Belfond.

RAYBAud, Antoine. (2005), "Saint-John Perse à l'irruption du nucléaire (l'impact sur Vents) », dans Henriette LEVILLAIN et Mireille SACotTE (dir.), Saint-John Perse (1945-1960). Une poétique pour l'âge nucléaire, Paris, Klincksieck, coll. "Bibliothèque contemporaine », p. 225-243. 
RobBe-GriLlet, Alain. (1957), La Jalousie, Paris, Minuit ;

—. (1963), Pour un nouveau roman, Paris, Minuit, coll. «Critique»;

—. (1970a), Projet pour une révolution à New York, Paris, Minuit ;

—. (1970b), encart de 4 pages (s. l. n. d.) inséré dans Projet pour une révolution à New York;

- (1972), intervention dans la discussion qui suit «Sur le choix des générateurs », dans Jean RICARDOU et Françoise VAN Rossum-GuYon (dir.), Nouveau Roman: hier, aujourd'hui, Paris, U.G.É., coll. « 10/18 », t. II, p. 163-173 ;

-. (1976), "Comment travaillent les écrivains ? Alain RobbeGrillet: "Chez moi, c'est la structure qui produit le sens" ", entretien avec Jean-Louis DE RAMBuRES. Le Monde des livres, 16 janvier : p. 17 ;

—. (1978a), Un régicide, Paris, Minuit ;

—. (1978b), "La Cible», préface au catalogue Jasper Johns, Paris, Centre Georges-Pompidou ;

-. (1978c), Traces suspectes en surface, West Islip, Universal Limited Art Editions (35 exemplaires connus) ;

—. (1984), Le Miroir qui revient, Paris, Minuit ;

—. (1990), Invasion blanche, Paris, La Différence ;

- . (1993), "A Graveyard of Identities and Uniforms », dans Judi FreEMAN, Mark Tansey, San Francisco, Chronicle Books, p. 7-11;

—. (1994), Les Derniers Jours de Corinthe, Paris, Minuit ;

-. (2001), La Reprise, Paris, Minuit. 
SAInT-John Perse. (1982), CEuvres complètes, Paris, Gallimard, coll. «Bibliothèque de la Pléiade».

SARRAUTE, Nathalie. (1949), Portrait d'un inconnu, Paris, Robert Marin ;

—. L'Ère du soupçon. (1956), Paris, Gallimard ;

—. Tropismes. (1957), Paris, Minuit.

Simon, Claude. (1957), Le Vent. Tentative de restitution d'un retable baroque, Paris, Minuit ;

—. (1959), « Cendre », Revue de Paris, mars, p. 79-82 ;

—. (1960a), La Route des Flandres, Paris, Minuit ;

—. (1960b), « Matériaux de construction », Les Lettres nouvelles, décembre, p. 112-122 ;

—. (1970), Orion aveugle, Genève, Skira, coll. « Les Sentiers de la création »;

—. (1971), Les Corps conducteurs, Paris, Minuit ;

—. (1975), Leçon de choses, Paris, Minuit ;

-. (1988a), Album d'un amateur, Remagen-Rolandseck, Rommerskirchen, coll. « Signatur »;

-. (1988b), "Attaques et stimuli (entretien inédit)», dans Lucien DÄLlEnBACH, Claude Simon, Paris, Seuil, coll. «Les Contemporains », p. 170-181;

—. (1989), « Claude Simon, "Et à quoi bon inventer ?" », propos recueillis par Marianne ALPHANT. Libération, $\mathrm{n}^{\circ} 2573$, 31 août, p. 14. 
STENDHAL. (1823), Racine et Shakespeare, chap. III : «Ce que c'est que le Romanticisme ».

VALÉRY, Paul. (1960), Regards sur le monde actuel, dans OEuvres complètes, Paris, Gallimard, coll. «Bibliothèque de la Pléiade », t. II.

Woolf, Virginia. (1923-1935), Freshwater. A Comedy.

\section{Résumé}

Nouveau, ça ne veut rien dire. Preuve en est la facilité avec laquelle a été détourné le sens originel de l'expression «nouveau roman». Avant-garde et modernité vont certes censément de pair : est nouveau ce qui n'est pas encore connu, ce qui étonne la réception, ce qui produit un changement radical. Les Amériques n'étaient pas l'horizon d'attente de Christophe Colomb et les lecteurs des années 1950 ne s'attendaient pas à la rénovation romanesque lancée rue Bernard-Palissy. Dans les deux cas, on n'avait rien vu venir, on ne savait pas ce qui arrivait, on ignorait vers quoi l'on se dirigeait, personne n'était vraiment préparé. Dans le champ littéraire de l'après-guerre, les perspectives étaient d'autant plus bouchées que l'horizon était recouvert des cendres d'Auschwitz et de celles de la bombe atomique. De là, en Europe, une littérature de la ruine, alors même que, de l'autre côté de l'Atlantique, les États-Unis sont un pôle d'attraction, géographique, historique et culturel, qui fascine tous ceux qui sont épris d'expérimentations et de liberté artistiques. C'est de là que les Nouveaux Romanciers vont prendre une envergure internationale et que plusieurs d'entre eux connaissent un regain d'inspiration, jusque même abandonner définitivement les terres connues de la narration. 